Vol. 43 (1991) [187-192]

\title{
ON QUASI-MONOTONE SEQUENCES AND THEIR APPLICATIONS
}

\section{HÜSEYIN BOR}

In this paper using $\delta$-quasi-monotone sequences a theorem on $\left|\bar{N}, p_{n}\right|_{k}$ summability factors of infinite series, which generalises a theorem of Mazhar [7] on $|C, 1|_{k}$ summability factors of infinite series, is proved. Also we apply the theorem to Fourier series.

\section{INTRODUCTION}

A sequence $\left(c_{n}\right)$ of positive numbers is said to be quasi-monotone if $n \Delta c_{n} \geqslant-\alpha c_{n}$ for some $\alpha>0$ and it is said to be $\delta$-quasi-monotone, if $c_{n} \rightarrow 0, c_{n}>0$ ultimately and $\Delta c_{n} \geqslant-\delta_{n}$, where $\left(\delta_{n}\right)$ is a sequence of positive numbers (see [1]).

Let $\sum a_{n}$ be a given infinite series with partial sums $\left(s_{n}\right)$. By $u_{n}$ and $t_{n}$ we denote the $n$th $(C, 1)$ means of the sequences $\left(s_{n}\right)$ and $\left(n a_{n}\right)$, respectively. The series $\sum a_{n}$ is said to be summable $|C, 1|_{k}, k \geqslant 1$, if (see [4])

$$
\sum_{n=1}^{\infty} n^{k-1}\left|u_{n}-u_{n-1}\right|^{k}<\infty
$$

But since $t_{n}=n\left(u_{n}-u_{n-1}\right)$ (see [6]), condition (1.1) can also be written as

$$
\sum_{n=1}^{\infty} \frac{1}{n}\left|t_{n}\right|^{k}<\infty
$$

Let $\left(p_{n}\right)$ be a sequence of positive numbers such that

$$
P_{n}=\sum_{v=0}^{n} p_{v} \rightarrow \infty \text { as } n \rightarrow \infty,\left(P_{-i}=p_{-i}=0, i \geqslant 1\right)
$$

The sequence-to-sequence transformation

$$
w_{n}=\frac{1}{P_{n}} \sum_{v=0}^{n} p_{v} s_{v}
$$

Received 29 March 1990

Copyright Clearance Centre, Inc. Serial-fee code: 0004-9729/91 \$A2.00+0.00. 
defines the sequence $\left(w_{n}\right)$ of the $\left(N, p_{n}\right)$ means of the sequence $\left(s_{n}\right)$ generated by the sequence of coefficients $\left(p_{n}\right)$ (see [5]). The series $\sum a_{n}$ is said to be summable $\left|\bar{N}, p_{n}\right|_{k}, k \geqslant 1$, if (see [2])

$$
\sum_{n=1}^{\infty}\left(P_{n} / p_{n}\right)^{k-1}\left|w_{n}-w_{n-1}\right|^{k}<\infty
$$

In the special case when $p_{n}=1$ for all values of $n$ (respectively $k=1$ ), then $\left|\bar{N}, p_{n}\right|_{k}$ summability is the same as $|C, 1|_{k}$ (respectively $\left|\bar{N}, p_{n}\right|$ ) summability. If we write

$$
X_{n}=\sum_{v=0}^{n} p_{v} / P_{v}
$$

then $\left(X_{n}\right)$ is a positive increasing sequence tending to infinity with $n$.

2.

Mazhar [7] has proved the following theorem for $|C, 1|_{k}$ summability factors by using $\delta$-quasi-monotone sequences.

THEOREM A. Let $\lambda_{n} \rightarrow 0$ as $n \rightarrow \infty$. Suppose that there exists a sequence of numbers $\left(A_{n}\right)$ which it is $\delta$-quasi-monotone with $\sum n \delta_{n} \log n<\infty, \sum A_{n} \log n$ is convergent and $\left|\Delta \lambda_{n}\right| \leqslant\left|A_{n}\right|$ for all $n$. If

$$
\sum_{n=1}^{m} \frac{1}{n}\left|t_{n}\right|^{k}=O(\log m) \text { as } m \rightarrow \infty,
$$

then the series $\sum a_{n} \lambda_{n}$ is summable $|C, 1|_{k}, k \geqslant 1$.

3.

The aim of this paper is to generalise Theorem A for $\left|\bar{N}, p_{n}\right|_{k}$ summability. Now we shall prove the following theorem.

THEOREM 1. Let $\lambda_{n} \rightarrow 0$ as $n \rightarrow \infty$ and let $\left(p_{n}\right)$ be a sequence of positive numbers such that

$$
P_{n}=O\left(n p_{n}\right) \text { as } n \rightarrow \infty .
$$

Suppose that there exists a sequence of numbers $\left(A_{n}\right)$ which is $\delta$-quasi-monotone with $\sum n X_{n} \delta_{n}<\infty, \sum A_{n} X_{n}$ is convergent and $\left|\Delta \lambda_{n}\right| \leqslant\left|A_{n}\right|$ for all $n$. If

$$
\sum_{n=1}^{m} \frac{p_{n}}{P_{n}}\left|t_{n}\right|^{k}=O\left(X_{m}\right) \text { as } m \rightarrow \infty,
$$

then the series $\sum a_{n} \lambda_{n}$ is summable $\left|\bar{N}, p_{n}\right|_{k}, k \geqslant 1$.

REMARK. It should be noted that if we take $p_{n}=1$ for all values fo $n$ (in this case $X_{n} \sim \log n$ ) in Theorem 1 , then we get Theorem A. 
4.

We need the following lemmas for the proof of Theorem 1.

LEmma . Under the conditions of Theorem 1, we have that

$$
\left|\lambda_{n}\right| X_{n}=O(1) \text { as } n \rightarrow \infty
$$

Proof: Since $\lambda_{n} \rightarrow 0$ as $n \rightarrow \infty$.

$$
\begin{aligned}
\left|\lambda_{n}\right| X_{n} & =X_{n}\left|\sum_{v=n}^{\infty} \Delta \lambda_{v}\right| \leqslant X_{n} \sum_{v=n}^{\infty}\left|\Delta \lambda_{v}\right| \\
& \leqslant \sum_{v=0}^{\infty} X_{v}\left|\Delta \lambda_{v}\right| \leqslant \sum_{v=0}^{\infty} X_{v}\left|A_{v}\right|<\infty .
\end{aligned}
$$

Hence $\left|\lambda_{n}\right| X_{n}=O(1)$ as $n \rightarrow \infty$.

Lemma 2. If $\left(A_{n}\right)$ is $\delta$-quasi-monotone with $\sum n X_{n} \delta_{n}<\infty$ and $\sum A_{n} X_{n}$ is convergent, then

$$
\begin{gathered}
m X_{m} A_{m}=O(1) \text { as } m \rightarrow \infty \\
\sum_{n=1}^{\infty} n X_{n}\left|\Delta A_{n}\right|<\infty
\end{gathered}
$$

The proof of Lemma 2 is similar to the proof of Theorems 1 and 2 of Boas [1, case $\gamma=1]$ and hence is omitted.

5.

Proof of Theorem 1: Let $\left(T_{n}\right)$ be the sequence of $\left(N, p_{n}\right)$ means of the series $\sum a_{n} \lambda_{n}$. Then, by definition, we have

$$
T_{n}=\frac{1}{P_{n}} \sum_{v=0}^{n} p_{v} \sum_{r=0}^{v} a_{r} \lambda_{r}=\frac{1}{P_{n}} \sum_{v=0}^{n}\left(P_{n}-P_{v-1}\right) a_{v} \lambda_{v} .
$$

Then, for $n \geqslant 1$, we get

$$
T_{n}-T_{n-1}=\frac{p_{n}}{P_{n} P_{n-1}} \sum_{v=1}^{n} P_{v-1} a_{v} \lambda_{v}=\frac{p_{n}}{P_{n} P_{n-1}} \sum_{v=1}^{n} \frac{P_{v-1} \lambda_{v}}{v} v a_{v}
$$


Applying Abel's transformation to the right hand side of (5.2), we have

$$
\begin{aligned}
& T_{n}-T_{n-1}=\frac{p_{n}}{P_{n} P_{n-1}} \sum_{v=1}^{n-1} \Delta\left(\frac{P_{v-1} \lambda_{v}}{v}\right) \sum_{r=1}^{v} r a_{r}+\frac{p_{n} \lambda_{n}}{n P_{n}} \sum_{v=1}^{n} v a_{v} \\
& =\frac{(n+1) p_{n} t_{n} \lambda_{n}}{n P_{n}}-\frac{p_{n}}{P_{n} P_{n-1}} \sum_{v=1}^{n-1} p_{v} t_{v} \lambda_{v} \frac{v+1}{v}+\frac{p_{n}}{P_{n} P_{n-1}} \sum_{v=1}^{n-1} P_{v} \Delta \lambda_{v} t_{v} \frac{v+1}{v} \\
& \quad+\frac{p_{n}}{P_{n} P_{n-1}} \sum_{v=1}^{n-1} P_{v} \lambda_{v+1} t_{v} \frac{1}{v}=T_{n, 1}+T_{n, 2}+T_{n, 3}+T_{n, 4}, \text { say. }
\end{aligned}
$$

To complete the proof of Theorem 1, by Minkowski's inequality, it is sufficient to show that

$$
\sum_{n=1}^{\infty}\left(P_{n} / p_{n}\right)^{k-1}\left|T_{n, r}\right|^{k}<\infty, \text { for } r=1,2,3,4
$$

First, we have

$$
\begin{aligned}
& \sum_{n=1}^{m}\left(P_{n} / p_{n}\right)^{k-1}\left|T_{n, 1}\right|^{k}=O(1) \sum_{n=1}^{m} \frac{\left|\lambda_{n}\right| p_{n}\left|t_{n}\right|^{k}}{P_{n}} \\
& =O(1) \sum_{n=1}^{m-1} \Delta\left|\lambda_{n}\right| \sum_{v=1}^{n} \frac{p_{v}}{P_{v}}\left|t_{v}\right|^{k}+O(1)\left|\lambda_{m}\right| \sum_{n=1}^{m} \frac{p_{n}}{P_{n}}\left|t_{n}\right|^{k} \\
& =O(1) \sum_{n=1}^{m-1}\left|\Delta \lambda_{n}\right| X_{n}+O(1)\left|\lambda_{m}\right| X_{m}=O(1) \sum_{n=1}^{m-1}\left|A_{n}\right| X_{n}+O(1)\left|\lambda_{m}\right| X_{m}=O(1)
\end{aligned}
$$

as $m \rightarrow \infty$, by virtue of the hypotheses and Lemma 1 .

Now applying Hölder's inequality, as in $T_{n, 1}$, we have that

$$
\begin{aligned}
& \sum_{n=2}^{m+1}\left(P_{n} / p_{n}\right)^{k-1}\left|T_{n, 2}\right|^{k} \\
& \quad=O(1) \sum_{n=2}^{m+1} \frac{p_{n}}{P_{n} P_{n-1}}\left\{\sum_{v=1}^{n-1} p_{v}\left|t_{v}\right|^{k}\left|\lambda_{v}\right|^{k}\right\} \times\left\{\frac{1}{P_{n-1}} \sum_{v=1}^{n-1} p_{v}\right\}^{k-1} \\
& =O(1) \sum_{v=1}^{m}\left|\lambda_{v}\right|^{k-1}\left|\lambda_{v}\right| p_{v}\left|t_{v}\right|^{k} \sum_{n=v+1}^{m+1} \frac{p_{n}}{P_{n} P_{n-1}} \\
& =O(1) \sum_{v=1}^{m}\left|\lambda_{v}\right| \frac{p_{v}}{P_{v}}\left|t_{v}\right|^{k}=O(1)
\end{aligned}
$$

as $m \rightarrow \infty$. 
Again, using the fact that $P_{v}=O\left(v p_{v}\right)$, by (3.1), we get

$$
\begin{aligned}
& \sum_{n=2}^{m+1}\left(P_{n} / p_{n}\right)^{k-1}\left|T_{n, 3}\right|^{k}=O(1) \sum_{n=2}^{m+1} \frac{p_{n}}{P_{n} P_{n-1}^{k}}\left\{\sum_{v=1}^{n-1} P_{v}\left|\Delta \lambda_{v}\right|\left|t_{v}\right|\right\}^{k} \\
& =O(1) \sum_{n=2}^{m+1} \frac{p_{n}}{P_{n} P_{n-1}^{k}}\left\{\sum_{v=1}^{n-1} v p_{v}\left|A_{v}\right|\left|t_{v}\right|\right\}^{k} \\
& =O(1) \sum_{n=2}^{m+1} \frac{p_{n}}{P_{n} P_{n-1}}\left\{\sum_{v=1}^{n-1}\left(v\left|A_{v}\right|\right)^{k} p_{v}\left|t_{v}\right|^{k}\right\} \times\left\{\frac{1}{P_{n-1}} \sum_{v=1}^{n-1} p_{v}\right\}^{k-1} \\
& =O(1) \sum_{v=1}^{m}\left(v\left|A_{v}\right|\right)^{k-1} v\left|A_{v}\right| p_{v}\left|t_{v}\right|^{k} \sum_{n=v+1}^{m+1} \frac{p_{n}}{P_{n} P_{n-1}} \\
& =O(1) \sum_{v=1}^{m} v\left|A_{v}\right| \frac{p_{v}}{P_{v}}\left|t_{v}\right|^{k} \\
& =O(1) \sum_{v=1}^{m-1} \Delta\left(v\left|A_{v}\right|\right) \sum_{r=1}^{v} \frac{p_{r}}{P_{r}}\left|t_{r}\right|^{k}+O(1) m\left|A_{m}\right| \sum_{v=1}^{m} \frac{p_{v}}{P_{v}}\left|t_{v}\right|^{k} \\
& =O(1) \sum_{v=1}^{m-1}\left|\Delta\left(v\left|A_{v}\right|\right)\right| X_{v}+O(1) m\left|A_{m}\right| X_{m} \\
& =O(1) \sum_{v=1}^{m-1} v X_{v}\left|\Delta A_{v}\right|+O(1) \sum_{v=1}^{m-1}\left|A_{v+1}\right| X_{v}+O(1) m\left|A_{m}\right| X_{m} \\
& =O(1) \text { as } m \rightarrow \infty,
\end{aligned}
$$

by virtue of the hypotheses and Lemma 2 .

Finally, using the fact that $P_{v}=O\left(v p_{v}\right)$, by (3.1), as in $T_{n, 1}$ we have that

$$
\begin{aligned}
& \sum_{n=2}^{m+1}\left(P_{n} / p_{n}\right)^{k-1}\left|T_{n, 4}\right|^{k} \leqslant \sum_{n=2}^{m+1} \frac{p_{n}}{P_{n} P_{n-1}^{k}}\left\{\sum_{v=1}^{n-1} \frac{P_{v}}{v}\left|\lambda_{n+1}\right|\left|t_{v}\right|\right\}^{k} \\
& =O(1) \sum_{n=2}^{m+1} \frac{p_{n}}{P_{n} P_{n-1}^{k}}\left\{\sum_{v=1}^{n-1}\left|\lambda_{v+1}\right| p_{v}\left|t_{v}\right|\right\}^{k} \\
& =O(1) \sum_{n=2}^{m+1} \frac{p_{n}}{P_{n} P_{n-1}}\left\{\sum_{v=1}^{n-1}\left|\lambda_{v+1}\right|^{k} p_{v}\left|t_{v}\right|^{k}\right\} \times\left\{\frac{1}{P_{n-1}} \sum_{v=1}^{n-1} p_{v}\right\}^{k-1} \\
& =O(1) \sum_{v=1}^{m}\left|\lambda_{v+1}\right|^{k-1}\left|\lambda_{v+1}\right| p_{v}\left|t_{v}\right|^{k} \sum_{n=v+1}^{m+1} \frac{p_{n}}{P_{n} P_{n-1}} \\
& =O(1) \sum_{v=1}^{m}\left|\lambda_{v+1}\right| \frac{p_{v}}{P_{v}}\left|t_{v}\right|^{k}=O(1)
\end{aligned}
$$


as $m \rightarrow \infty$. Therefore, we get

$$
\sum_{n=1}^{m}\left(P_{n} / p_{n}\right)^{k-1}\left|T_{n, r}\right|^{k}=O(1) \text { as } m \rightarrow \infty, \quad \text { for } r=1,2,3,4
$$

This completes the proof of Theorem 1.

6.

Let $f(t)$ be a periodic function with period $2 \pi$ and integrable $(L)$ over $(-\pi, \pi)$. Let

$$
f(x) \simeq \sum_{n=0}^{\infty} A_{n}(x), \phi(t)=\frac{1}{2}\{f(x+t)+f(x-t)\} \text { and } \phi_{1}(t)=\frac{1}{t} \int_{0}^{t} \phi(u) d u
$$

It is well known that if $\phi_{1}(t) \in B V(0, \pi), t_{n}(x)=O(1)$, where $t_{n}(x)$ is the $n$th $(C, 1)$ mean of the sequence $\left(n A_{n}(x)\right)$ (see [3]). Hence, using this fact, we get the following result for Fourier series.

TheOREM 2. If $\phi_{1}(t) \in B V(0, \pi)$ and the sequences $\left(p_{n}\right),\left(\lambda_{n}\right)$ and $\left(X_{n}\right)$ satisfy the conditions of Theorem 1 , then the series $\sum A_{n}(x) \lambda_{n}$ is summable $\left|\bar{N}, p_{n}\right|_{k}, k \geqslant 1$.

\section{REFERENCES}

[1]. R.P. Boas (Jr.), 'Quasi-positive sequences and trigonometric series', Proc. Lond. Math. Soc. 14(A) (1965), 38-46.

[2] H. Bor, 'On two summability methods', Math. Proc. Camb. Philos. Soc. 97 (1985), 147-149.

[3] K.K. Chen, 'Functions of bounded variation and Cesàro means of Fourier series', Acad. Sinica Sc. Records 1 (1954), 283-289.

[4] T.M. Flett, 'On an extension of absolute summability and some theorems of Littlewood and Paley', Proc. Lond. Math. Soc. 7 (1957), 113-141.

[5] G.H. Hardy, Divergent Series (Oxford University Press, 1949).

[6] E. Kogbetliantz, 'Sur les séries absolument sommables par la méthode des moyennes arithmétiques', Bull. Sci. Math. 49 (1925), 234-256.

[7] S.M. Mazhar, 'On generalized quasi-convex sequence and its applications', Indian J. Pure Appl. Math. 8 (1977), 784-790. 